\title{
Mesh Plug Technique for Umbilical and Paraumbilical Hernias Repair; A Novel Technique
}

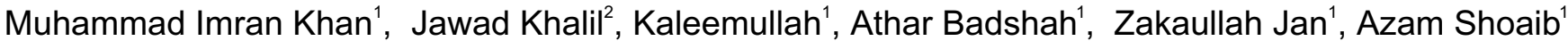

ABSTRACT

Background: Umbilical and paraumbilical herniae are the most common ventral herniae occurring at or around the umbilical ring. Umbilical hernia accounts for almost $6-10 \%$ of all abdominal wall herniae. A ventral hernia is abnormal protrusion of peritoneal sac through the musculo-aponeurotic abdominal wall defect. Females are affected five times more as compared to males. The various risk factors include obesity, chronic cough and constipation, ascites and pregnancy in females.

Objective: To determine the outcome of mesh plug repair of umbilical and paraumbilical hernias in terms of operative time and postoperative complications.

Material and Methods: This study was conducted at surgical department of Khyber Teaching Hospital, Peshawar from $1^{\text {st }}$ January 2012 to $31^{\text {st }}$ December 2016. Total of 131 patients with umbilical and Para umbilical hernias were studied. Patients were operated using mesh plug technique and operative time and postoperative complications were recorded.

Results: There were 91 female patients compared to 40 male patients (M/F ratio 2.2:1). The overall mean age was $41 \pm 19.3$ years. The mean operative time was $23 \pm 9.2$ minutes. In the early postoperative period 3 patients had superficial surgical site infection. Seroma was observed in 2 patients. Mesh plug infection was observed in 1 patient while chronic pain was observed in 2 patients. None of the patients had recurrence on 24 months follow up.

Conclusion: Mesh plug for small umbilical and paraumbilical hernias in adults is a safe, convenient, fast and minimally invasive technique with a lower rate of postoperative complications and recurrence.

Keywords: Mesh plug, umbilical hernia, paraumbilical hernia, recurrence.

This article may be cited as: Khan IM, Khalil J, Kaleemullah, Badshah A, Jan Z, Shoaib A.

Mesh Plug Technique for Umbilical and Paraumbilical Hernias Repair; A Novel Technique. J Saidu Med Coll Swat. 2020; 10(1):27-30

\section{INTRODUCTION}

Umbilical and paraumbilical herniae are the most common ventral herniae occurring at or around the umbilical ring. ${ }^{1}$ Umbilical hernia accounts for almost $6-10 \%$ of all abdominal wall herniae. ${ }^{2,3} \mathrm{~A}$ ventral hernia is abnormal protrusion of peritoneal sac through the musculo-aponeurotic abdominal wall defect. Females are affected five times more as compared to males. The various risk factors include obesity, chronic cough and constipation, ascites and pregnancy in females. ${ }^{4}$

The contents of hernial sac may vary such as omentum or gut which underlies its varied clinical presentations. Clinically most patients present to outpatient department with umbilical deformity/lump or pain in or around the umbilicus. Some can present with obstructive symptoms like abdominal pain, vomiting, fever and distension. ${ }^{5-7}$ Ventral hernia repair is a common surgical procedure with both laparoscopic and open techniques being in use. In open surgery traditionally suture repair and prosthetic mesh repair are the options ${ }^{8}$. Mesh repair is the optimal

\footnotetext{
1. Department of Surgical A Unit, KTH, MTI, Peshawar

2. Pak International Medical College, Peshawar

Correspondence: Dr. Muhammad Imran Khan

Flat \# 6, Nursing Flats, Khyber Teaching Hospital, Peshawar.

Email: drimranmarwat82@gmail.com

Cell Number: 0333-9889959
}

choice for hernia defects more than $3 \mathrm{~cm}$ in size. There is, however, much debate as to what should be an ideal way of treating hernia defects up to $3 \mathrm{~cm}$ in size. ${ }^{9}$ In this study we have used a new technique of putting a mesh plug for ventral hernia repair having defect size of $=3 \mathrm{~cm}$.

\section{MATERIALAND METHODS}

The present study was conducted at Khyber Teaching Hospital, Peshawar from $1^{\text {st }}$ January 2012 to $31^{\text {st }}$ December 2016 as part of a single center prospective study. Total duration of study was five years. The study was approved from the Ethical Committee of the institution.

In this study 131 patients with umbilical and paraumbilical hernias who presented to outpatient department were included. The diagnosis was made clinically. The patients were selected through consecutive non probability sampling technique.

All patients with clinical diagnosis of reducible umbilical and Para-umbilical hernias with defect size of $\leq 3 \mathrm{~cm}$, having age between 18 to 60 years, American Society of Anesthesiologists Class I,II (ASA-I,II) and willing to do the follow-up protocol were included in this study. Patients with recurrent hernias, hernias with defect size of more than $3 \mathrm{~cm}$, complicated hernias and patients having comorbidities were excluded from the study. 
All the patients fulfilling the inclusion criteria were admitted a day before surgery, as part of the routine protocol of our unit. History, physical examination and routine investigations for anesthesia fitness such as complete blood count, blood sugar level, Hepatitis B and C screening, chest radiography and electrocardiography of the patients with age above 50 years were performed. The patients were explained the risks and benefits of the procedure. Verbal and written informed consent was obtained. The patients were operated by a same consultant surgeon under general anesthesia. The patients were given a prophylactic dose of third-generation cephalosporin at induction as part of the protocol.

In patients having umbilical hernia an infra or supra-umbilical semilunar incision was made while transverse incision, almost $4 \mathrm{~cm}$ in length, was utilized in para-umbilical hernias. The skin was incised down to the subcutaneous fat and fascia and the defect was identified and hernia sac was dissected out. After isolation of the sac it was opened near the neck and contents, if any, were returned to the peritoneal cavity. The opening in the sac was closed with vicryl $2 / 0$ and reduced back into the abdominal cavity. In case of large sacs the redundant sac was partially removed at the fundus and the remaining sac was closed with running vicryl $2 / 0$ suture and reduced. A $6 \times 11 \mathrm{~cm}$ Prolene mesh was rolled and folded (cigarette stub) to tailor it to the size of the hernia ring and was pushed down through the hernia defect. The mesh was anchored to the margin of the defect all around using continuous prolene $2 / 0$ suture. The subcutaneous tissue was closed with $3 / 0$ absorbable suture and skin was closed with $2 / 0$ prolene suture in subcuticular continuous fashion with vacuum drainage for only one day.

The patients were not allowed orally until they were fully recovered from anesthesia. Patients were discharged once they were able to tolerate diet and had a good pain control. A standardized questionnaire was used to record the data.

All the operative details were recorded. The operative time was recorded in minutes. Pain was measured qualitatively (subjectively) using visual analog scale with $0 \mathrm{~mm}$ representing no pain and $100 \mathrm{~mm}$ representing unbearable pain. The length of hospital stay was determined as the number of nights spent at the hospital postoperatively.
Postoperative complications were recorded in the proforma during the hospital stay and till 2 years (scheduled follow-up visit at 10 days after surgery and additional visits six monthly in OPD). Telephone interviews were recorded when patients did not return for follow-up. Wound infection was defined as redness, purulent or sero-purulent discharge from the incision site observed within 30 days postoperatively. Fat liquefaction or Seroma was defined as yellow or pale brown exudates from the wound on $3^{\text {rd }}$ to $10^{\text {th }}$ postoperative day with localized swelling and no inflammatory manifestations such redness, heat and pain. Hematoma was defined as swelling without inflammatory signs and observation of clotted blood after opening the skin incision. Recurrence was defined as reappearance of the lump or a defect of the midline aponeurosis at the operated site. Confounding variables were controlled through strictly following the exclusion criteria.

The data was analyzed using statistical package for social sciences SPSS, version 20. Continuous variables, such as age, hospital stay and operative duration, were presented as mean \pm SD, while categorical variables, such as gender and postoperative complication, were expressed with frequency and percentages using 95\% confidence interval.

\section{RESULTS}

In this study 131 patients were operated upon using mesh plug technique and included in the final analysis. There were 91 female patients compared to 40 male patients (M/F ratio 2.2:1). The overall mean age was $41 \pm 19.3$ years, as shown in Table 1. The mean operative time was $23 \pm 9.2$ minutes. The mean diameter of the hernial defect was $1.7 \pm 1.1 \mathrm{~cm}$. The mean hospital stay was $24 \pm 11$ hours.

The postoperative complications are shown in Table 2. In the early postoperative period 3 patients had superficial surgical site infection. Seroma was observed in 2 patients. Mesh plug infection was observed in 1 patient while chronic pain was observed in 2 patients. None of the patients had recurrence on 24 months follow up. 
Table 1. Demographic and clinical characteristics

\begin{tabular}{|c|c|}
\hline Variables & $\mathrm{N}(\%)$ or Mean \pm SD \\
\hline Gender & $91(69)$ \\
\hline Male & $40(31)$ \\
\hline Female & $41 \pm 19.3$ \\
\hline Age (Years) & $38 \pm 17$ \\
\hline Overall & $44 \pm 15.4$ \\
\hline Male &
\end{tabular}

Table 2. Complications after mesh plug repair.

\begin{tabular}{|c|c|}
\hline Complications & $\mathrm{N}(\%)$ \\
\hline Immediate complications & $3(2.2)$ \\
\hline Surgical site infection & $2(1.5)$ \\
\hline Seroma & $1(0.76)$ \\
\hline Fat liquefaction & $0(0)$ \\
\hline Hematoma & $0(0)$ \\
\hline Abscess & $1(0.76)$ \\
\hline Long term complications & $2(1.5)$ \\
\hline Mesh infection & $0(0)$ \\
\hline Chronic pain & \\
\hline Recurrence & \\
\hline
\end{tabular}

\section{DISCUSSION}

A variety of techniques are utilized to repair umbilical and paraumbilical hernias, with simple suture repair to complex laparoscopic hernioplasties being the two extremes. Conventional repairs are associated with high recurrence rates while laparoscopic techniques require a sophisticated setup and long learning curve. Open mesh repair is the middle pathway being especially very popular for larger defects $(>3 \mathrm{~cm}$ in diameter) because of their low recurrence rates, faster recovery and decreased postoperative pain. There are advocates favoring prosthetic repairs even for smaller hernias less than $3 \mathrm{~cm}$ as facial or primary repairs often fail and lead to recurrence in significant proportion of patients. $^{10-14}$

The present study shows that the overall mean age was $41 \pm 19.3$ years. This is corroborated by other studies. ${ }^{5,15}$ There are others who showed that these hernias maximally in the sixth decade of life which is in contrast to our findings. ${ }^{16,17}$ In our study females were affected more than males with a male to female ratio of $2.2: 1$. This is consistent with results of others. ${ }^{18}$ other studies, however, showed conflicting results with females amounting to almost $80 \%$ of the studied population., ${ }^{5,17}$
Operative duration remains a much talked about aspect among experts whenever comparing procedures. The mean operative time in our study was $23 \pm 9.2$ minutes. This is consistent with others who reported almost similar results. ${ }^{18}$ Tollens $T$ demonstrated significantly longer duration of surgery compared to our findings ${ }^{12}$. All the procedures in our study were performed by a consultant surgeon with sufficient surgery load and the difference can be explained by the expertise and case load.

According to Johnson, a new procedure must have, in addition to other benefits, such as less operative time, ease in performance and the advantage of safety (less complications and morbidity). ${ }^{19}$ In this study the overall complication rate was quite low. Wound infections may not be a serious complication as such but can cause inconvenience to the patient, impacting convalescence and quality of life. In the present study, only 3 patients developed wound infection (superficial surgical site infection). Similar observations are reported from other national and international studies. ${ }^{5,18}$ None of the patients developed abscess or hematoma in the present study. Less subcutaneous tissue dissection to expose only the sac and ring may be the reason for rarity of these complications. 
Mesh plug infection was observed in one patient. This finding is reproduced by other investigators as well. ${ }^{5,17,18}$ The only patient who developed mesh plug infection was advised specific antibiotic according to culture and sensitivity report with a favorable outcome without resorting plug removal.

On long term follow up we found only 2 patients with chronic pain. This finding is mirrored in other studies investigating the role of mesh plug $^{18}$.

During follow up we observed that none of our patients had recurrence. Xie $\mathrm{Y}$, et al in an observational study found appreciated recurrence in $2(1.9 \%)$ out of 123 patients utilizing mesh plug technique for umbilical hernia repair which is consistent with our results ${ }^{18}$. Others, however, reported considerably higher recurrence rate in contrast to our findings ${ }^{9}$. Our results suggest that the use of mesh plug for small umbilical and paraumbilical hernia contributes to lower recurrence rates.

\section{CONCLUSION}

Mesh plug for small umbilical and paraumbilical hernias in adults is a safe, convenient, fast and minimally invasive technique with a lower rate of complications, less postoperative complication, chronic pain, and a lower recurrence rate.

\section{REFERENCES}

1. Schumpelick V, Fitzgibbons RJ. Recurrent hernia. Springer-Verlag Berlin Heidelberg; 2007.

2. Aslani N, Brown CJ. Does mesh offer an advantage over tissue in the open repair of umbilical hernias? A systematic review and meta-analysis. Hernia. 2010 Oct 1;14(5):455-62.

3. Brancato G, Privitera A, Gandolfo L, Donati M, Caglià $P$. Plug-technique for umbilical hernia repair in the adult. Minerva chirurgica. 2002 Feb;57(1):13-6..

4. Dabbas N, Adams K, Pearson K, Royle GT. Frequency of abdominal wall hernias: is classical teaching out of date?. JRSM short reports. 2011 Jan;2(1):1-6..

5. Saeed N, Khan MS, syed SA. Comparison of complications between mayo's repair and mesh repair (pre-peritoneal approach) for para-umbilical hernia. The management of myopic macular hole without retinal detachment. 2005 Oct; 10:38.

6. Agrawal VP, Shetty NS, Narasimhaprasad A. Obstructed Umbilical Hernia: A Normal Presentation with Abnormal Contents. Euroasian journal of hepatogastroenterology. $2015 \mathrm{Jul} ; 5(2): 110$..

7. Johnstone JM, Rintoul RF. Abdominal Hernia in Farquharson's Text Book of Operative Surgery. Rintoul RF "Churchill Livingstone. 1995:523-31.

8. Christoffersen MW, Helgstrand F, Rosenberg J, Kehlet $\mathrm{H}$, Strandfelt $\mathrm{P}$, Bisgaard $\mathrm{T}$. Long-term recurrence and chronic pain after repair for small umbilical or epigastric hernias: a regional cohort study. The American Journal of Surgery. 2015 Apr 1;209(4):725-32.

9. Venclauskas L, Jokubauskas M, Zilinskas J, Zviniene $\mathrm{K}$, Kiudelis M. Long-term follow-up results of umbilical hernia repair. Videosurgery and Other Miniinvasive Techniques. 2017 Dec;12(4):350.

10. Halm JA, Heisterkamp J, Veen HF, Weidema WF. Long-term follow-up after umbilical hernia repair: are there risk factors for recurrence after simple and mesh repair. Hernia. 2005 Dec 1;9(4):334-7.

11. Venclauskas L, Šilanskaitè J, Kiudelis M. Umbilical hernia: factors indicative of recurrence. Medicina. 2008;44(11):855.

12. Tollens $\mathrm{T}$, Den Hondt $\mathrm{M}$, Devroe K, Terry C, Speybroeck S, Aelvoet C, Vanrykel JP. Retrospective analysis of umbilical, epigastric, and small incisional hernia repair using the Ventralex ${ }^{\mathrm{TM}}$ hernia patch. Hernia. 2011 Oct 1;15(5):531-40.

13. Sanjay P, Reid TD, Davies EL, et al. Retrospective comparison of mesh and sutured repair for adult umbilical hernias. Hernia 2005;9:1.

14. Christoffersen, M.W., Helgstrand, F., Rosenberg, J., Kehlet, H. and Bisgaard, T., 2013. Lower reoperation rate for recurrence after mesh versus sutured elective repair in small umbilical and epigastric hernias. A nationwide register study. World journal of surgery, 37(11), pp.2548-2552..

15. Qamaruddin B. Historical background a study of repair of paraumbilical hernia. Karachi 1990:40.

16. Arroyo A, Garcia P, Perez F, Andreu J, Candela F, Calpena R. Randomized clinical trial comparing suture and mesh repair of umbilical hernia in adults. British journal of surgery. 2001 Oct;88(10):1321-3.

17. Sebastian AA, Perez F, Serrano P, Costa D, Oliver I, Ferrer R, Lacueva J, Calpena R. Is prosthetic umbilical hernia repair bound to replace primary herniorrhaphy in the adult patient?. Hernia. 2002 Dec 1;6(4):175-7..

18. Xie Y, Song Y, Ma D, Jian F, Zhang S, Lu A, WangY, Lei W. Retrospective analysis of smaller than $3-\mathrm{cm}$ umbilical hernia repair with the lightweight macroporous mesh. Medicine. 2018 Sep;97(36).

19. Johnson A. Laparoscopic surgery. The Lancet. 1997 Mar 1;349(9052):631-5. 